\title{
The outcome of a temporomandibular joint compression test for the diagnosis of arthralgia is confounded by concurrent myalgia
}

\author{
Anna Lövgren ${ }^{1}$ (D) C Corine M. Visscher ${ }^{2}$ - Per Alstergren ${ }^{3,4,5}$ • Frank Lobbezoo ${ }^{2}$ - Birgitta Häggman-Henrikson ${ }^{1,3}$. \\ Anders Wänman ${ }^{1}$
}

Received: 17 December 2018 / Accepted: 2 April 2019 /Published online: 25 April 2019

(C) The Author(s) 2019

\begin{abstract}
Objective Pain in the orofacial region may originate from different structures, and one challenge for the clinician is to determine the primary origin of pain reported by the patient. In clinical practice, it is important to discriminate between a temporomandibular joint (TMJ) pain disorder and jaw muscle pain; therefore, tests that are proposed for such purposes warrant evaluation. The aim of the present study was to evaluate the outcome of a TMJ compression test in relation to a Diagnostic Criteria for Temporomandibular Disorders (DC/TMD) arthralgia diagnosis.

Methods A study population $(n=300)$, randomly selected from the adult population in Västerbotten, Sweden, was examined according to the DC/TMD criteria and with a TMJ compression test. This test is comprised of forceful unilateral biting for $20 \mathrm{~s}$ on a wooden spatula in the first molar region. Familiar pain on the contralateral side to the clenching side was considered a positive test outcome.

Results Positive contralateral outcome of the TMJ compression test was associated with an arthralgia diagnosis $(B=1.737$; OR 5.7, 95\% CI 3.3-9.9). This association was confounded by concurrent myalgia $(B=1.737 \rightarrow B=0.996,42.7 \%)$.

Conclusion In a general population, a negative TMJ compression test was strongly associated with the absence of a contralateral TMJ arthralgia diagnosis according to DC/TMD. The association between a positive TMJ compression test and a DC/TMD arthralgia diagnosis was confounded by the presence of myalgia.

Clinical relevance Concurrent myalgia renders the usefulness of the TMJ compression test for predicting an arthralgia diagnosis questionable.
\end{abstract}

Keywords Temporomandibular disorder · Temporomandibular joint · DC/TMD $\cdot$ Diagnostic tests · Diagnostic accuracy

Anna Lövgren

anna.lovgren@umu.se

1 Department of Clinical Oral Physiology, Faculty of Medicine, University of Umeå, 90187 Umeå, Sweden

2 Department of Oral Kinesiology, Academic Centre for Dentistry Amsterdam (ACTA), University of Amsterdam and Vrije Universiteit, Amsterdam, The Netherlands

3 Department of Orofacial Pain and Jaw function, Faculty of Odontology, Malmö University, Malmö, Sweden

4 Specialized Pain Rehabilitation, Skåne University Hospital, Lund, Sweden

5 Scandinavian Centre for Orofacial Neurosciences, Malmö, Sweden

\section{Introduction}

Temporomandibular disorders (TMD) are considered musculoskeletal disorders that embrace pain or dysfunction of the temporomandibular joint (TMJ) and masticatory muscles [1, 2]. TMD is the most common reason for chronic pain in the orofacial region [3] and is commonly associated with interference in daily life $[4,5]$.

The Diagnostic Criteria for Temporomandibular Disorders (DC/TMD) is a valid process to diagnose the most common TMD conditions. In the DC/TMD, both TMJ pain (arthralgia) and myalgia diagnoses are based on self-reported pain modified by function, in combination with familiar pain elicited during jaw movements or palpation [6]. Both these diagnoses have a high diagnostic sensitivity and specificity. In the clin- 
ical setting, it is important to discriminate between a TMJ pain disorder and jaw muscle pain since both the etiology and the management of these conditions may differ. A discriminating diagnostic test is therefore warranted in order to recognize arthralgia and myalgia independently. This can be of special clinical relevance when the arthralgia is related to arthritis. In such case, anti-inflammatory pharmacological management may be considered as a primary treatment regimen in order to reduce inflammatory activity, to reduce pain, and to prevent further degeneration of the cartilage and bone tissue in the TMJ [7].

We previously reported a substantial overlap between TMJ arthralgia and masticatory myalgia in a validation study of three screening questions (3QTMD) in relation to DC/TMD diagnoses [7]. These findings are in line with those reported by Schiffman and co-workers [8]. In the clinical examination to reach the diagnosis arthralgia, palpation of the lateral pole affects also tissues outside the TMJ proper that includes muscle tissue, skin, and connective tissue. Moreover, mandibular movements probably do not cause solely TMJ pain but also muscle pain, given that the muscles are activated. The location of pain, especially in patients with chronic pain, is an uncertain indicator for its origin due to pain spreading, referred pain, and sensitization mechanisms [9]. Even though pain location may still be relevant for an arthralgia diagnosis, the close location of the lateral pterygoid and masseter muscles in relation to the TMJ complicates the diagnostics. In addition, local or generalized hyperalgesia may lead to positive signs from palpation of jaw muscles and/or the TMJ and thereby affect the outcome of the diagnostic procedure. There is thus a need for evaluating diagnostic tests that are hypothesized to more precisely determine if the origin of pain is located to the muscles, to the TMJ, or to both.

One such diagnostic test may be a TMJ compression test [10]. In this test, the patient bites hard on a wooden spatula placed between the teeth in the molar region on one side in order to physically compress intraarticular structures, especially on the contralateral side. A previous experimental study showed that biting on a rigid interference placed unilaterally in the molar region caused a frontal, upward rotation of the mandibular condyle contralateral to the interference in all subjects [11]. A recent study reported that patients with unilateral TMJ disc displacement had a higher prevalence of elicited contralateral TMJ pain when biting on a bite force transducer in the molar region [12]. Unilateral clenching in the molar region has been shown to induce a significant reduction of the TMJ joint space contralateral to the biting side [13]. These studies indicate that unilateral clenching in the molar region induces a compression of the contralateral TMJ, and may thus elicit a nociceptive response if the intraarticular tissues are sensitized.

In addition to contralateral TMJ pain, it was also suggested that unilateral clenching can provoke ipsilateral fatigue and pain in the masticatory muscles [14]. In clinical practice, ipsilateral pain during unilateral clenching may, therefore, be indicative for myalgia of the masticatory muscles. However, the association between myalgia and ipsilateral pain during a TMJ compression test has not been evaluated. An evaluation of the TMJ compression test can thus add relevant diagnostic information.

The first aim of this study was to determine if the TMJ compression test could predict a DC/TMD arthralgia or myalgia diagnosis. The second aim was to determine possible confounders of such associations. Our hypothesis was that contralateral elicited familiar pain would be predictive for a TMJ pain condition (arthralgia), and that an ipsilaterally elicited familiar pain would be predictive for a masticatory muscle pain condition (myalgia).

\section{Materials and methods}

\section{Participants}

The study population comprised 300 adult individuals (98 men and 202 women; mean age 38.7 years, SD 13.8). Participants were randomly recruited from the Public Dental Health Care in the county of Västerbotten, Sweden, based on their answers to three screening questions for TMD (3Q/ TMD) [15]. The 3Q/TMD is likely to be positive in the case of DC/TMD pain diagnoses and negative when DC/TMD pain diagnoses are absent. In total, 148 screen positives and 152 screen negatives were included in the study. The entire recruitment process has been previously described in detail [15].

\section{Diagnostic criteria for temporomandibular disorders diagnoses of arthralgia and myalgia}

All participants underwent the standardized DC/TMD Axis I examination (questionnaire and clinical examination) The DC/TMD diagnoses "arthralgia" and "myalgia" were used as reference standards [6]. The DC/TMD pain diagnoses are based on self-reported pain during the last 30 days that is modified (i.e., aggravated or relieved) by jaw function. During the clinical examination, an arthralgia diagnosis is confirmed if the patient reports pain in the TMJ area during the last 30 days, as confirmed by the examiner, in combination with familiar pain from the TMJ on jaw movements or palpation. A myalgia diagnosis is confirmed if the patient reports pain in the masseter or temporal muscles during the last 30 days in combination with familiar pain with either muscle palpation or maximum jaw opening. Familiar pain was defined as "pain that is similar or like the pain [the patient] may have had in that same part of your body in the last 30 days" as per the definition in the DC/TMD [6]. All examinations were performed by a single examiner (author AL) who 
was calibrated [16] and was blinded as to whether the participant screened positive to the $3 \mathrm{Q} / \mathrm{TMD}$ or not. The reliability of the examiner was assessed at a DC/TMD Training and Calibration Centre and was found to be "almost perfect" for arthralgia and myalgia [17] (Cohen's Kappa values; arthralgia right, 0.87; arthralgia left, 0.88; myalgia, 0.82).

\section{TMJ compression test}

The TMJ compression test was performed with the participant biting as hard as possible for $20 \mathrm{~s}$ on a double-wooden spatula (thickness $2 \times 2 \mathrm{~mm}$ ) in the first permanent molar region (Fig. 1). The participant was allowed to refrain from or abort the test if it became too uncomfortable due to, for example, tooth pain. The participant was instructed to report experiences and locations of familiar pain on the ipsilateral or contralateral side in the orofacial area during the test period. In total, 12 tests on the right side and 16 tests on the left side could not be performed due to missing teeth or to the test being too uncomfortable for the participant.

To determine if an association between a positive test outcome and an arthralgia diagnosis was confounded [18], the following variables were evaluated: myalgia, limited maximum jaw opening ( $<40 \mathrm{~mm}$ including vertical overbite), and widespread pain. The association between a positive test outcome and a myalgia diagnosis was evaluated with arthralgia, limited vertical maximum opening $(<40 \mathrm{~mm}$ including vertical overbite), and widespread pain as possible confounders.

Widespread pain was assessed with the use of the body drawing in the McGill Pain Questionnaire [19]. The whole body was divided into 19 sites, and widespread pain was categorized as positive when pain was reported in seven or more of these sites [20].

\section{Statistical analysis}

Frequencies for positive test outcomes are presented descriptively. Since all individuals could contribute with data from two tests, sensitivity and specificity were calculated for each test separately. The sensitivities and specificities were therefore calculated for a positive contralateral compression test outcome in relation to a DC/TMD arthralgia diagnosis, and for a positive ipsilateral compression test outcome in relation to a myalgia diagnosis, respectively. Confidence intervals for sensitivity and specificity were calculated according to the Wilson score method [21]. To account for the two measurements in each individual in the study sample $(n=300)$, the association between a positive test outcome as the dependent variable and the DC/TMD diagnosis as the independent variable was established using an unstructured, binary logistic generalized estimating equation (GEE).

To evaluate if an association was confounded, the following models were analyzed:

Model I:1 TMJ compression test (contralateral side) vs. arthralgia

Model I:2 Model I:1 + myalgia

Model I:3 Model I:2 + limited jaw maximum opening

Model I:4 Model 1:3 + widespread pain

Model II:1 TMJ compression test (ipsilateral side) vs. myalgia

Model II:2 Model 1:1 + arthralgia

Model II:3 Model II:2 + limited jaw maximum opening

Model II:4 Model II:3 + widespread pain

The association between the TMJ compression test and arthralgia or myalgia, respectively, was considered to be

Fig. 1 Biomechanical hypothesis behind the temporomandibular joint compression test

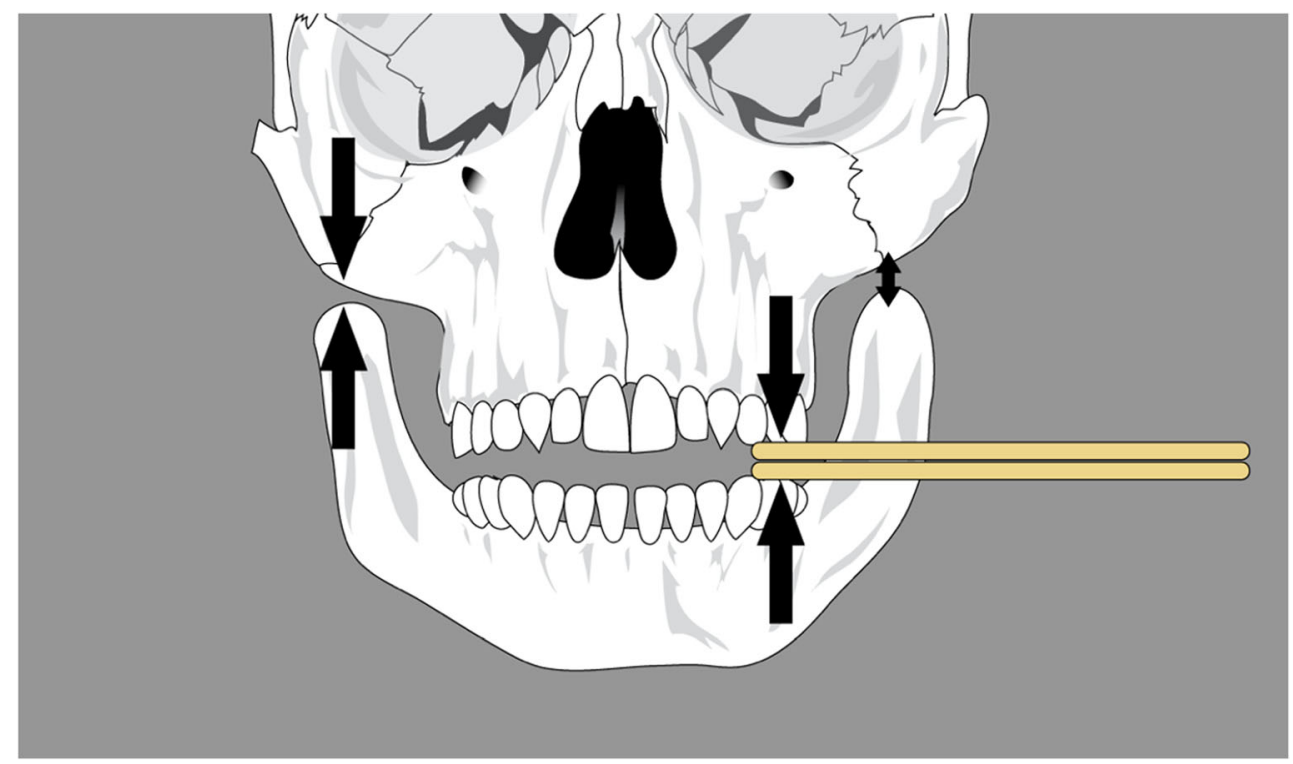


confounded when the regression coefficient of the TMJ compression test changed by $>10 \%$ as compared with its value in the previous model (without that confounding variable) [22].

To check for multicollinearity, all possible independent variables were entered in a correlation matrix. If the correlation coefficient between two independent variables exceeded 0.70 , multicollinearity [18], which may disrupt the model, was considered to be present. In that case, the independent variable that is the most strongly associated variable will be chosen to enter into the binary logistic model. The statistical analyses were carried out with SPSS version 24.002 and a probability level of $<0.05$ was considered statistically significant.

The study was approved by the Regional Ethical Review Board at Umeå University, Sweden (reference number 2012331-31M). All participants signed a written informed consent, prior to the data collection. As recommended for diagnostic accuracy studies, the STARD statement was followed [23].

\section{Results}

In total, 60 participants (20\% of the study population) had a positive compression test outcome on the contralateral side of the clenching ( 36 on the right side, and 45 on the left TMJ side) (Table 1). In addition, the test provoked an ipsilateral familiar pain outcome in 68 participants (51 times on the right side and 49 times on the left side).

A DC/TMD arthralgia diagnosis was established in 66 individuals (Table 1), representing $22 \%$ of the study population (13 men and 53 women, mean age 38.1 years; SD 15.0). Three individuals (1\%) qualified for an arthralgia diagnosis without concurrent myalgia.

The sensitivity and specificity for a positive outcome of the TMJ compression test on the contralateral side in relation to an arthralgia diagnosis on the contralateral side were 0.40 (95\% CI $0.31-0.50)$ and 0.91 (95\% CI $0.88-0.94)$, respectively (Table 2). The sensitivity and specificity for an ipsilateral familiar pain in relation to a myalgia diagnosis were 0.35 (95\%

Table 1 Frequency distribution in the study sample $(n=300)$

\begin{tabular}{ll}
\hline & $n(\%)$ \\
\hline DC/TMD arthralgia diagnosis & $66(22)$ \\
Unilateral right & $18(6)$ \\
Unilateral left & $9(3)$ \\
Bilateral & $39(13)$ \\
Myalgia & $106(35)$ \\
TMJ compression test positive (contralateral) & $60(20)$ \\
TMJ compression test positive (ipsilateral) & $68(23)$ \\
Limited jaw opening & $13(4)$ \\
Widespread pain $\geq 7 / 19$ sites & $94(31)$ \\
\hline
\end{tabular}

Table 2 Sensitivity and specificity for contralateral compression test in relation to TMJ contralateral arthralgia according to the DC/TMD (TMJ sides, $n=572 *)$

Arthralgia No arthralgia

TMJ compression test (contralateral) positive $0.40(40) \quad 0.09$ (41)

TMJ compression test (contralateral) negative 0.60 (59) 0.91 (432)

*Missing data from 28 tests

CI $0.29-0.42$ ) and 0.93 (95\% CI $0.90-0.95)$, respectively (Table 3).

The positive contralateral outcome of the TMJ compression test was associated with a contralateral arthralgia diagnosis $(B=1.737$; OR $5.7,95 \%$ CI 3.3-9.9) (Table 4). This association was confounded by concurrent myalgia $(B=1.737 \rightarrow$ $B=0.996,42.7 \%$ ), but not by widespread pain or limitation of vertical jaw movements. Ipsilateral positive test outcomes were associated with a myalgia diagnosis $(B=1.962$; OR 7.1 95\% CI 3.9-13.0) (Table 5). This association was confounded by a concurrent ipsilateral arthralgia diagnosis $(B=1.962 \rightarrow$ $B=1.270,35.2 \%)$ and widespread pain $(B=1.280 \rightarrow B=$ $1.003,21.6 \%$ ), but not by the limitation of vertical jaw movements.

\section{Discussion}

The main finding from this study is that the TMJ compression test has a high specificity and it generates a high negative predictive value for contralateral DC/TMD diagnosis of arthralgia. This indicates that a negative TMJ compression test is strongly associated with the absence of a contralateral TMJ arthralgia diagnosis according to DC/TMD. On the other hand, a positive outcome of the TMJ compression test is only modestly associated with contralateral TMJ arthralgia diagnosis. In addition, the association between the TMJ compression test and a contralateral TMJ arthralgia diagnosis is confounded by the presence of myalgia. This indicates that the predictive value of the TMJ compression test for a diagnosis of contralateral TMJ arthralgia is even lower in the case of concurrent myalgia.

In clinical practice, the outcome of a diagnostic test should preferably provide reliable and valid guidance for the selection of specific treatment strategies. For any pain condition,

Table 3 Sensitivity and specificity for ipsilateral compression test in relation to myalgia according to the DC/TMD (TMJ sides, $n=572 *$ )

\begin{tabular}{lll}
\hline & Myalgia & No myalgia \\
\hline TMJ compression test (ipsilateral) positive & $0.35(73)$ & $0.07(27)$ \\
TMJ compression test (ipsilateral) negative & $0.65(133)$ & $0.93(339)$ \\
\hline
\end{tabular}

*Missing data from 28 tests 
Table 4 Binary logistic, generalized estimating equations for associations between positive compression test and contralateral DC/TMD arthralgia expressed as regression coefficients (B), odds ratio (OR), and its $95 \%$ confidence intervals within parenthesis

\begin{tabular}{|c|c|c|c|c|c|c|c|c|}
\hline & \multicolumn{2}{|c|}{$\begin{array}{l}\text { Contralateral arthralgia } \\
\text { Single association }\end{array}$} & \multicolumn{2}{|c|}{ Model $1+$ myalgia } & \multicolumn{2}{|c|}{ Model $2+$ limited vertical movement } & \multicolumn{2}{|c|}{ Model 3 + widespread pain } \\
\hline & $B$ & OR & $B$ & OR & $B$ & OR & $B$ & OR \\
\hline TMJ compression test positive & 1.737 & $5.7(3.3-9.9)$ & 0.996 & $2.7(1.5-5.0)$ & 0.954 & $2.6(1.4-4.8)$ & 0.880 & $2.4(1.3-4.4)$ \\
\hline
\end{tabular}

early identification and intervention are important to prevent chronicity [24]. Chronic pain conditions also tend to embrace more complex and widespread symptoms. Therefore, in these conditions, specific diagnostic strategies are required in order to distinguish a local symptom from a widespread condition. Since TMJ pain and masticatory muscle pain may, at least in part, have different etiologies, there is a clinical need for a diagnostic test that accurately distinguishes these conditions. However, the findings from the present study indicate a low sensitivity, when only a minority of the individuals with TMJ arthralgia report a positive TMJ compression test outcome. With regard to the potential confounding factors, myalgia was found to influence the association between the test outcomes and the diagnosis of arthralgia. There may be multiple possible explanations. Firstly, the results may indicate that the DC/TMD criteria do not sufficiently discriminate myalgia from arthralgia, which may be supported by the fact that 95\% of those with arthralgia also fulfilled the criteria for myalgia. Secondly, it may indicate that myalgia and arthralgia are in fact concurrent conditions and mirror a local, regional, or generalized sensitization. The complexity of the temporomandibular region means that any diagnostic test may be hampered by the presence of comorbidity [25].

The ipsilateral positive test outcome also showed a moderate diagnostic precision for the detection of myalgia with low sensitivity and high specificity. In clinical practice, the absence of an ipsilateral positive test outcome may thus be indicative for the absence of myalgia. Since the association with myalgia was not only confounded by a concurrent arthralgia but also by widespread pain, this may indicate that myalgia could be related to general hyperalgesia rather than to local factors [26]. This finding indicates that general pain conditions may be incorrectly interpreted as a local TMD pain condition.
Taken together, the results suggest that the TMJ compression test is associated with the absence of TMJ arthralgia if there is a negative outcome of the test, even after the correction of possible confounders. Future studies should explore whether the addition of the compression test to the test battery of the DC/TMD would improve the sensitivity and specificity of the current DC/TMD algorithms.

\section{Methodological considerations}

The study population was randomly selected from the Public Dental health care and therefore, the results may be considered as representative for the adult populations in general, in Sweden and for comparable countries. In addition, all clinical examinations were performed by a formally trained and calibrated examiner which accounts for high diagnostic reliability. However, the study was primarily developed for investigating the TMJ compression test as part of the clinical examination. As a consequence, the index test (the compression test) was not performed ahead of the reference standard test (the DC/TMD), as proposed in the STARD initiative [23]. The DC/TMD examination could thereby have provoked pain that is then reproduced during the index test for this study, i.e., the TMJ compression test. Another possible limitation is that the pain location after the TMJ compression test was not assessed for the TMJ and masseter/temporalis muscles separately. In this case, these factors would have caused false positive responses. However, the low sensitivity contradicts this possible shortcoming.

\section{Conclusion}

In a general population, a negative TMJ compression test was strongly associated with the absence of a contralateral TMJ arthralgia diagnosis according to DC/TMD. The association

Table 5 Binary logistic, generalized estimating equations for associations between ipsilateral TMJ compression positive test outcome, and myalgia expressed as regression coefficients (B), odds ratio (OR), and its $95 \%$ confidence intervals within parenthesis

\begin{tabular}{|c|c|c|c|c|c|c|c|c|}
\hline & \multicolumn{2}{|c|}{$\begin{array}{l}\text { Myalgia } \\
\text { single association }\end{array}$} & \multicolumn{2}{|c|}{ Model $1+$ arthralgia } & \multicolumn{2}{|c|}{ Model $2+$ limited vertical movement } & \multicolumn{2}{|c|}{ Model 3 + widespread pain } \\
\hline & $B$ & OR & $B$ & OR & $B$ & OR & $B$ & OR \\
\hline TMJ compression test positive & 1.962 & $7.1(3.9-13.0)$ & 1.270 & $3.6(1.8-7.0)$ & 1.276 & $3.6(1.8-7.1)$ & 1.003 & $2.7(1.3-5.7)$ \\
\hline
\end{tabular}


between a positive TMJ compression test and a DC/TMD arthralgia diagnosis was confounded by the presence of a DC/TMD myalgia diagnosis. Concurrent myalgia renders the usefulness of the TMJ compression test for predicting an arthralgia diagnosis questionable.

Funding Financial support was provided through a regional agreement between Umeå University and Västerbotten County Council in cooperation with the field of Medicine, Odontology and Health and the Swedish Dental Society.

\section{Compliance with ethical standards}

Conflict of interest The authors declare that they have no conflicts of interest.

Ethical approval All procedures performed in studies involving human participants were in accordance with the ethical standards of the institutional and/or national research committee and with the 1964 Helsinki declaration and its later amendments or comparable ethical standards.

Open Access This article is distributed under the terms of the Creative Commons Attribution 4.0 International License (http:// creativecommons.org/licenses/by/4.0/), which permits unrestricted use, distribution, and reproduction in any medium, provided you give appropriate credit to the original author(s) and the source, provide a link to the Creative Commons license, and indicate if changes were made.

\section{References}

1. Okeson JP (2013) Management of temporomandibular disorders and occlusion. 7th edn. Mosby

2. Dworkin SF, LeResche L (1992) Research diagnostic criteria for temporomandibular disorders: review, criteria, examinations and specifications, critique. J Craniomandib Disord 6(4):301-355

3. Lipton JA, Ship JA, Larach-Robinson D (1993) Estimated prevalence and distribution of reported orofacial pain in the United States. J Am Dent Assoc 124(10):115-121

4. LeResche L (1997) Epidemiology of temporomandibular disorders: implications for the investigation of etiologic factors. Crit Rev Oral Biol Med 8(3):291-305

5. Rollman A, Visscher CM, Gorter RC, Naeije M (2012) Care seeking for orofacial pain. J Orofac Pain 26(3):206-214

6. Schiffman E, Ohrbach R, Truelove E, Look J, Anderson G, Goulet JP, List T, Svensson P, Gonzalez Y, Lobbezoo F, Michelotti A, Brooks SL, Ceusters W, Drangsholt M, Ettlin D, Gaul C, Goldberg LJ, Haythornthwaite JA, Hollender L, Jensen R, John MT, De Laat A, de Leeuw R, Maixner W, van der Meulen M, Murray GM, Nixdorf DR, Palla S, Petersson A, Pionchon P, Smith B, Visscher CM, Zakrzewska J, Dworkin SF, International Rdc/Tmd Consortium Network IafDR, Orofacial Pain Special Interest Group International Association for the study of Pain (2014) Diagnostic criteria for temporomandibular disorders (DC/ TMD) for clinical and research applications: recommendations of the International RDC/TMD Consortium Network* and Orofacial Pain Special Interest Group†. J Oral Facial Pain Headache 28 (1):627. https://doi.org/10.11607/jop.1151

7. Cairns BE (2010) Pathophysiology of TMD pain-basic mechanisms and their implications for pharmacotherapy. J Oral Rehabil 37(6):391-410. https://doi.org/10.1111/j.1365-2842.2010.02074.x
8. Schiffman EL, Truelove EL, Ohrbach R, Anderson GC, John MT, List T, Look JO (2010) The research diagnostic criteria for temporomandibular disorders. I: overview and methodology for assessment of validity. J Orofac Pain 24(1):7-24

9. Mense S (2003) The pathogenesis of muscle pain. Curr Pain Headache Rep 7(6):419-425

10. van Eijden TM (2000) Biomechanics of the mandible. Crit Rev Oral Biol Med 11(1):123-136

11. Rassouli NM, Christensen LV (1995) Experimental occlusal interferences. Part III. Mandibular rotations induced by a rigid interference. J Oral Rehabil 22(10):781-789

12. Kumazaki Y, Kawakami S, Hirata A, Oki K, Minagi S (2016) Ipsilateral molar clenching induces less pain and discomfort than contralateral molar clenching in patients with unilateral anterior disc displacement of the temporomandibular joint. J Oral Facial Pain Headache 30(3):241-248. https://doi.org/10.11607/ofph.1405

13. Kuboki T, Azuma Y, Orsini MG, Takenami Y, Yamashita A (1996) Effects of sustained unilateral molar clenching on the temporomandibular joint space. Oral Surg Oral Med Oral Pathol Oral Radiol Endod 82(6):616-624

14. Sforza C, Zanotti G, Mantovani E, Ferrario VF (2007) Fatigue in the masseter and temporalis muscles at constant load. Cranio 25(1): 30-36. https://doi.org/10.1179/crn.2007.006

15. Lövgren A, Visscher CM, Häggman-Henrikson B, Lobbezoo F, Marklund S, Wänman A (2016) Validity of three screening questions (3Q/TMD) in relation to the DC/TMD. J Oral Rehabil 43(10): 729-736. https://doi.org/10.1111/joor.12428

16. Vilanova LS, Garcia RC, List T, Alstergren P (2015) Diagnostic criteria for temporomandibular disorders: self-instruction or formal training and calibration? J Headache Pain 16:505. https://doi.org/ 10.1186/s10194-015-0505-9

17. McHugh ML (2012) Interrater reliability: the kappa statistic. Biochem Med (Zagreb) 22(3):276-282

18. Field A (2013) Discovering statistics using IBM SPSS Statistics. 4th edn. SAGE

19. Melzack R (1975) The McGill Pain Questionnaire: major properties and scoring methods. Pain 1(3):277-299

20. Wolfe F, Clauw DJ, Fitzcharles MA, Goldenberg DL, Katz RS, Mease P, Russell AS, Russell IJ, Winfield JB, Yunus MB (2010) The American College of Rheumatology preliminary diagnostic criteria for fibromyalgia and measurement of symptom severity. Arthritis Care Res 62(5):600-610. https://doi.org/10.1002/acr.20140

21. Newcombe RG (1998) Two-sided confidence intervals for the single proportion: comparison of seven methods. Stat Med 17(8):857-872

22. Lee PH (2014) Is a cutoff of $10 \%$ appropriate for the change-inestimate criterion of confounder identification? J Epidemiol 24(2): 161-167

23. Bossuyt PM, Reitsma JB, Bruns DE, Gatsonis CA, Glasziou PP, Irwig LM, Lijmer JG, Moher D, Rennie D, de Vet HC (2003) Standards for reporting of diagnostic accuracy. Towards complete and accurate reporting of studies of diagnostic accuracy: the STARD initiative. BMJ 326 (7379):41-44

24. Macfarlane GJ (2016) The epidemiology of chronic pain. Pain 157(10): 2158-2159. https://doi.org/10.1097/j.pain.000000000000676

25. Koutris M, Visscher CM, Lobbezoo F, Naeije M (2013) Comorbidity negatively influences the outcomes of diagnostic tests for musculoskeletal pain in the orofacial region. Pain 154(6):927932. https://doi.org/10.1016/j.pain.2013.03.004

26. Plesh O, Wolfe F, Lane N (1996) The relationship between fibromyalgia and temporomandibular disorders: prevalence and symptom severity. J Rheumatol 23(11):1948-1952

Publisher's note Springer Nature remains neutral with regard to jurisdictional claims in published maps and institutional affiliations. 